\title{
Comparison of Unilateral and Bilateral
}

\section{Oxidation of Iron*}

\section{By Shigeji TANIGUCHI** and D.L. CARPENTER***}

\begin{abstract}
Synopsis
An attempt has been made to compare the characteristics of unilateral and bilateral oxidation of thin iron coupon specimens at $800^{\circ} \mathrm{C}$ in purified oxygen at atmospheric pressure. It is believed that unilateral oxidation created bending moments increasing the plastic flow of the oxide scales and thus improving scale/substrate adherence.

As a result of this, the rate of unilateral oxidation was found, in general, to be higher than that of bilateral oxidation.

It has been suggested that relief of oxide growth stresses due to the enhanced plastic flow of the oxide scale during the unilateral oxidation may modify diffusion through the oxide layer.
\end{abstract}

\section{Introduction}

The importance of oxide growth stresses, oxide plasticity and the fate of vacancies transported to the scale/metal interfaces in determining the growth characteristics of p-type semiconducting oxides on a range of metals at relatively high temperatures has been pointed out by several workers. ${ }^{1-6)}$ The present authors $^{7)}$ have also reported that oxidation behaviour of cold-rolled Remko iron coupons, involving deviations from a simple parabolic relationship, in a temperature range $700^{\circ}$ to $900^{\circ} \mathrm{C}$ can be accounted for by considering the above factors.

It follows that if oxide scales are plastic enough to relieve growth stresses and consequently to eliminate the interfacial voids formed at the scale/substrate interface by the coalescence ${ }^{6,8)}$ of excess vacancies, good adherence of oxide scales with the substrate may be maintained.

However, the complete relief of the stresses can hardly be expected, since very high temperatures are required for appreciable plastic flow of the scale and reasonable strain rate to take place. ${ }^{9)}$ Therefore, it may be said that growing oxide scales are generally in stressed state.

On the other hand, several measurements ${ }^{10-14)}$ of stresses developed during oxidation of a range of metals and alloys have been carried out using " bending strip " methods in which one side of a thin strip specimen was oxidized, the other side being protected with a coating material.

The moment created by such unilateral oxidation bends the specimen, while bilateral oxidation does not, since stresses developed on both sides of the specimen balance, although slight dilations ${ }^{5,15)}$ might be expected.

Therefore, it may be said that the unilateral oxidation can create a favourable situation for plastic flow of a growing oxide scale, which may influence oxida- tion kinetics and scale adhesion by changing scale/ substrate interface characteristics, if specimens are thin enough to be distorted by the moment created.

In order to examine this concept the present study was carried out. This also comprises a part of the work $^{16)}$ concerned with measurement of stresses developed during the oxidation of Remko iron using a "bending strip" method.

The result of the work indicated that the following three sets of stresses were mainly operative during oxidation of iron forming a three layered oxide scale composed of the outermost hematite, intermediate magnetite and the innermost wustite layers:

a) Tensile stresses in the substrate iron due to vacancy injection into the substrate and corresponding compressive stresses in the wustite layer at the wustite/iron interface

b) Compressive stresses in the wustite layer due to wustite formation from magnetite involving a volume increase and corresponding tensile stresses in the magnetite layer at the magnetite/wustite interface

c) Compressive stresses in the magnetite layer due to magnetite formation from hematite involving a volume increase and corresponding tensile stresses in the hematite layer at the hematite/magnetite interface.

Deformation mechanism of a range of growing oxides under ordinary oxidation conditions has been reported $^{5)}$ to be predominantly Nabarro-Herring creep and dislocation glide in the oxides under the conditions is very limited. ${ }^{9)}$

Here, the words "bilateral oxidation " are used in order to emphasize the distinction between unilateral oxidation and a normal (bilateral) oxidation in which both sides of a coupon specimen are oxidized.

\section{Experimental}

Coupon specimens measuring $20 \times 10 \times 0.3 \mathrm{~mm}$ thick were cut from cold rolled strip $(70 \%$ reduction in thickness) of Remko iron which contains the following impurities in ppm by weight; C 300, Si 100, Mn 120, S 100, Cr 50, Ni 50, Cu 100, Sn 20, O 40 and $\mathrm{N}$ 100. Specimens were then wet ground on a series of emery papers up to 600, wheel polished and electropolished in a solution composed of one part of perchloric acid and ten parts of glacial acetic acid at a current density of $0.2 \mathrm{~A} / \mathrm{cm}^{2}$ for $1.5 \mathrm{~min}$, the temperature being maintained below $25^{\circ} \mathrm{C}$.

Fully annealed specimens were prepared by heating

* Received March 25, 1977.

** Department of Metallurgical Engineering, Faculty of Engineering, Osaka University, Yamadakami, Suita, Osaka 565.

*** Department of Metallurgy and Materials Science, University College, Cardiff, Wales, U.K. 
at $1000^{\circ} \mathrm{C}$ for $4 \mathrm{~h}$ under a vacuum of $10^{-6}$ torr followed by furnace cooling under the same degree of vacuum.

One side of the specimen used for unilateral oxidation was coated with electroless nickel.

The plating solution was prepared by mixing $70 \mathrm{ml}$ of "Nifoss base solution No. 3747 " and $200 \mathrm{ml}$ of "Nifoss initial solution No. 3748 " (both produced by W. Canning and Co. Ltd.) with distilled water to make up of the final volume of $1 l$. The deposit from this solution contains $7 \%$ phosphorus by weight. One side of the specimen was protected with a "Lacomit" stopping off medium during the plating of the other side, the temperature being kept between $85^{\circ}$ and $90^{\circ} \mathrm{C}$. The $\mathrm{pH}$ value of the solution did not change during plating and remained at a value of 5 as shown by a $\mathrm{pH}$ indicator paper. Fresh solution was used for each operation, since any change in the solution composition results ${ }^{17}$ ) in change of both the reaction rate and nature of the deposit. The thickness of the deposit was measured intermittently and adjusted to about $27 \mu \mathrm{m}$, usually obtained after $3 \mathrm{~h}$.

After the coating operation, the coupon was annealed at $700^{\circ} \mathrm{C}$ for $1 \mathrm{~h}$ in a mixture of nitrogen $-5 \%$ hydrogen or under vacuum in order to stabilize the coating and to improve the reproducibility of the data. This treatment allowed recrystallization of cold-rolled specimen, subsequently referred to as recrystallized, the grain size was still much smaller than that of the fully annealed specimen. ${ }^{18)}$ Vickers hardness values of the coating were reported ${ }^{19)}$ to be 17 at $600^{\circ} \mathrm{C}$ and 3 at $800^{\circ} \mathrm{C}$, indicating its high ductility at these temperatures, thus permitting the bending of the unilaterally oxidizing specimens.

The coupon specimens used for bilateral oxidation for comparison were treated in a similar way since similar pre-treatments have been reported ${ }^{20,21)}$ to affect oxidation kinetics.

After these treatments the uncoated iron surfaces were again electropolished and etched in a solution of $0.5 \%$ hydrochloric acid in ethanol for a period of $2 \mathrm{~min}$ immediately prior to oxidation.

Oxidation runs were carried out at $800^{\circ} \mathrm{C}$ in purified oxygen flowing $400 \mathrm{ml} / \mathrm{min}$ at atmospheric pressure using a nichrome bifilial wound furnace equipped in an autorecording thermobalance.

At the end of the test the furnace was raised allowing the specimen being cooled rapidly in air.

Morphologies of oxide scales and substrate surfaces were examined using a scanning electron microscope and an optical microscope.

Diffusion of the coating material into the substrate during oxidation was examined employing a microanalyser technique.

The conditions for the above pre-treatments are summarized in Table 1 which includes oxidation of cold-rolled specimen oxidized without any treatment for reference purposes (No. 5 in Table 1), the oxidation behaviour of which has been reported elsewhere. ${ }^{\text {?) }}$ Further details of the experimental procedures have been reported elsewhere. ${ }^{18)}$

\section{Results}

\section{Kinetics}

Results of oxidation kinetics are expressed by weight gain per unit area of iron surface and the reproducibility of the data by vertical lines drawn through each kinetic curve which represents mean values of three runs under identical conditions. Comparison of oxidation kinetic behaviours of unilateral and bilateral oxidation with the specified metallurgical pre-treatments is shown in Fig. 1. The kinetic curve for a cold-rolled specimen oxidized bilaterally without any treatment is also shown in the figure for reference.

Curves 1 and 2 show a similar weight gain up to around $10 \mathrm{~min}$ in a near parabolic manner and after this period the weight gain of the unilateral oxidation is much larger than that of the bilateral oxidation which is followed by very small weight gain. Curves 3 and 4 also show a similar weight gain up to $30 \mathrm{~min}$ and after this period the unilateral oxidation shows larger weight gain than the bilateral oxidation. However, the weight gain of unilateral oxidation of recrystallized specimen is much greater than that of the fully annealed specimen.

Oxidation kinetic studies of specimens coated with electroless nickel on both sides, reported elsewhere, ${ }^{18}$ ) have shown that the weight gain by the oxidation of the coating material is $0.8 \mathrm{mg} / \mathrm{cm}^{2}$ after $1000 \mathrm{~min}$ at

Table 1. Conditions for the oxidation

\begin{tabular}{|c|c|c|c|c|}
\hline No. & $\begin{array}{c}\text { First } \\
\text { treatment }\end{array}$ & $\begin{array}{l}\text { Coat- } \\
\text { ing }\end{array}$ & Second treatment & \\
\hline 1 & \multirow{2}{*}{ Cold-rolled } & $\mathrm{C}$ & \multirow{2}{*}{$\begin{array}{l}\text { Annealed at } 700^{\circ} \mathrm{C} \\
\text { for } 1 \mathrm{~h} \text { in } \mathrm{N}_{2}-5 \% \mathrm{H}_{2}\end{array}$} & \multirow{5}{*}{$\begin{array}{l}\text { Oxidized in } \\
\text { purified } \\
\text { oxygen at } \\
800^{\circ} \mathrm{C}\end{array}$} \\
\hline 2 & & $\mathrm{~N}$ & & \\
\hline 3 & \multirow{2}{*}{$\begin{array}{l}\text { Fully } \\
\text { annealed }\end{array}$} & $\mathrm{C}$ & $\begin{array}{l}\text { Vacuum annealed } \\
\text { at } 700^{\circ} \mathrm{C} \text { for } 1 \mathrm{~h}\end{array}$ & \\
\hline 4 & & $\mathrm{~N}$ & - & \\
\hline 5 & Cold-rolled & $\mathrm{N}$ & - & \\
\hline
\end{tabular}

C: One side is coated with electroless nickel.

N : Non-coated

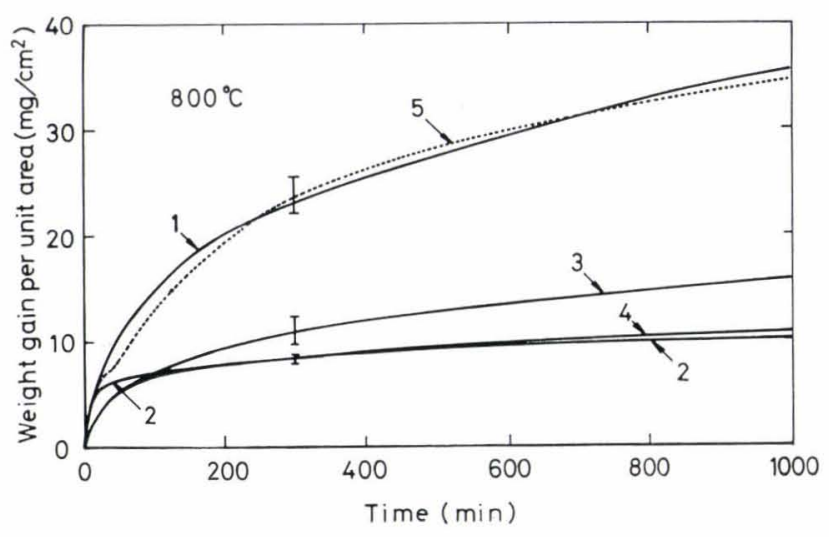

Fig. 1. Comparison of kinetic behaviours of unilateral and bilateral oxidation of Remko iron in purified oxygen at $800^{\circ} \mathrm{C}$ with the specified pre-treatments. Numbers in the figure correspond to those in the Table 1. 
$800^{\circ} \mathrm{C}$

This weight gain is two or three orders of magnitude smaller than that of the iron and is the same order of magnitude for nickel oxidation. ${ }^{22,23)}$

This can also be understood from Photo. 1, a cross section of the specimen oxidized under condition 1 , showing that the thickness of the oxide layer formed on the coating (bottom) is much smaller than that formed on iron (upper half).

The error introduced by neglecting the weight gain due to the oxidation of the coating material used for the unilateral oxidation was found to be less than $5.4 \%$.

Scales formed on the specimens oxidized under conditions 2 to 4 were very thin as expected from the kinetic results. Slight shock readily exfoliated these scales after cooling to room temperature, indicating their poor adherence to the substrates.

\section{Microscopic Studies}

Oxide scales formed on iron surface by the unilateral oxidation of recrystallized specimen showed a three layered structure composed of the thin outermost hematite, intermediate magnetite, and the innermost wustite layers, as Photo. 1.

However, it should be noted that the thickness ratio of wustite to magnetite to hematite was around 40 to 10 to 1 . This is quite different from the reported values ${ }^{24,25)}$ of around 95 to 5 to 1 obtained under ordinary oxidation.

Concentration profiles of iron, nickel, and phosphorus along a cross section of the above specimen are shown in Photo. 2. During the oxidation nickel and phosphorus diffused into iron substrate resulting in the final thickness of the diffusion zone about $10 \mu \mathrm{m}$. The iron profile shows that the oxide formed

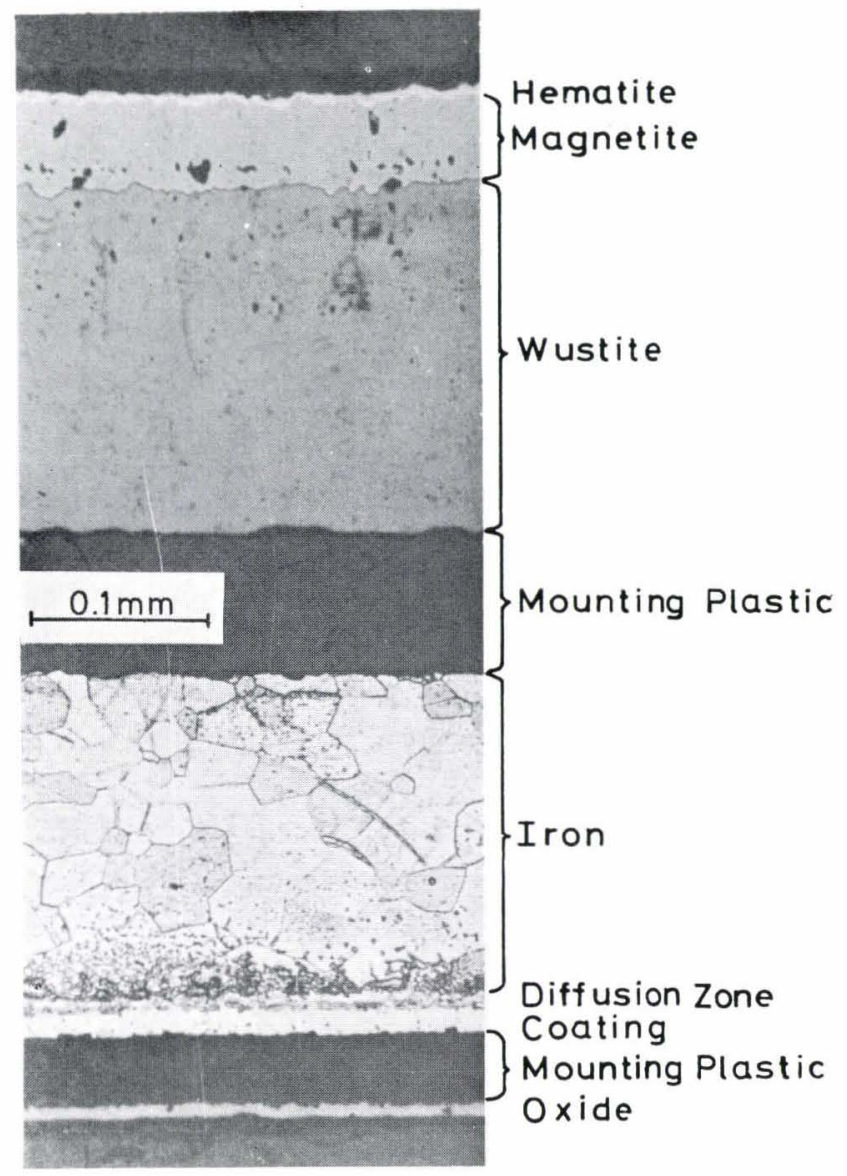

Photo. 1. Cross section of the specimen after 1000 min oxidation under condition 1 , showing a three layered oxide scale (composed of the outermost thin hematite, intermediate magnetite and the innermost wustite) formed on iron, very thin oxide formed on the coating and diffusion zone in the substrate a
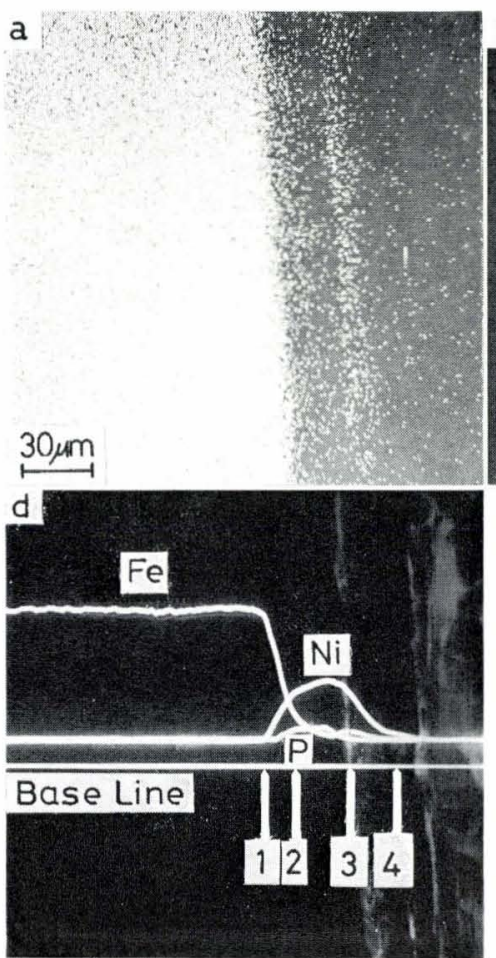
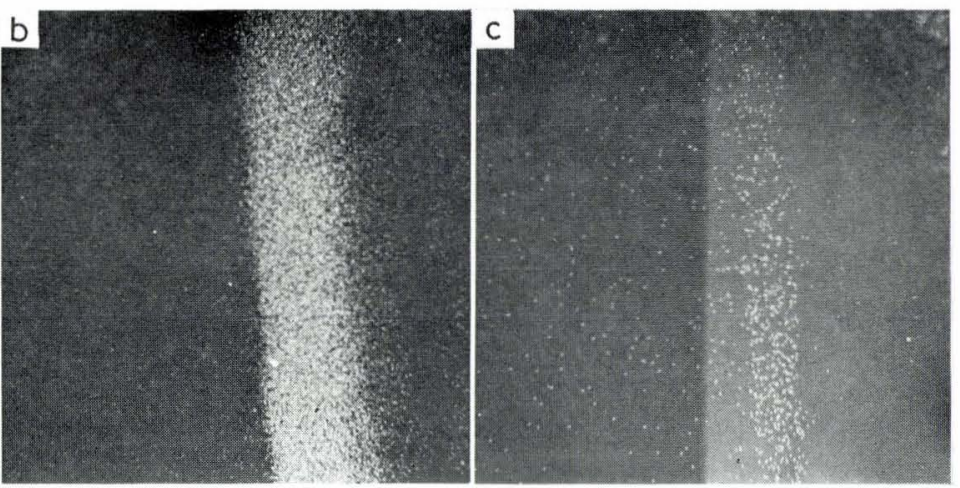

\section{1- 2 Diffusion Zone in Iron}

2 Original Iron/Electroless Nickel Interface

\section{2-3 Electroless Nickel Layer}

$3-4$ Oxide

Photo. 2. $\mathrm{K}_{\alpha}$-images of iron (a), nickel (b) and phosphorus (c), and their profiles across a cross section of the specimen (d) after oxidation under condition 1 
on the coating contains a small amount of iron, but it mainly composed of nickel oxide as shown by the nickel profile.

In general, substrate surfaces of the unilaterally oxidized specimens were corrugated as shown in Photo. 3 (a), while those of the bilaterally oxidized specimens were relatively smooth except where socalled oxide bridges were found, Photo. 3 (b). Difference in local surface concentrations of these oxide bridges created a patchwork pattern on the substrate surface.

The unilateral oxidation of the fully annealed specimens produced less corrugated substrate surfaces, Photo. 3 (c). In contrast to this, relatively large ridges were formed on grain boundaries of the substrate surface, while the rest of the surface was relatively smooth during the bilateral oxidation of the fully annealed specimen as shown in Photo. 3 (d). The oxide fracture took place along lines corresponding to these oxide ridges after cooling to room temperature.

Smoothing of the substrate surface between these oxide bridges or ridges is considered to be a result of annealing after the area lost contact. Further, these corrugated substrate surfaces are characteristic for unilateral oxidation.

The oxide scales formed by the unilateral oxidation of recrystallized specimens were composed of columnar wustite, magnetite, and thin hematite layers as shown in Photo. 4 (a). This structure is similar to those formed during the oxidation of cold-rolled specimens without any pre-treatment, as shown in Photo. 5, except the thickness ratios of the oxide layers. On the other hand, partial transformation of wustite from columnar to an equiaxed structure took place during the unilateral oxidation of the fully annealed specimens, while virtually complete transformation of wustite and magnetite layers occurred during the bilateral oxidation of the fully annealed specimens, Photo. 4 (b).

Oxidation behaviour under condition 5 is characterized by a depression appeared around $30 \mathrm{~min}$ and the subsequent recovery in the oxidation curve (Fig. 1). This has been attributed ${ }^{7}$ to a temporary void formation at the scale/substrate interface followed by the subsequent elimination of the voids by plastic flow of wustite resulted from compressive stresses developed by the mechanisms (a) and (b). After this recovery the scale/substrate contact was maintained. However, the substrate surface was not corrugated nor smoothed as shown in Photo. 5 and oxide thickness ratio was also different from that under condition 1 .

\section{Discussion}

It has been reported ${ }^{24-26)}$ that oxidation of iron in oxygen or air at atmospheric pressure at $800^{\circ} \mathrm{C}$ follows a nearly parabolic relationship, the rate determining mechanism being mainly mass transport through the wustite layer.
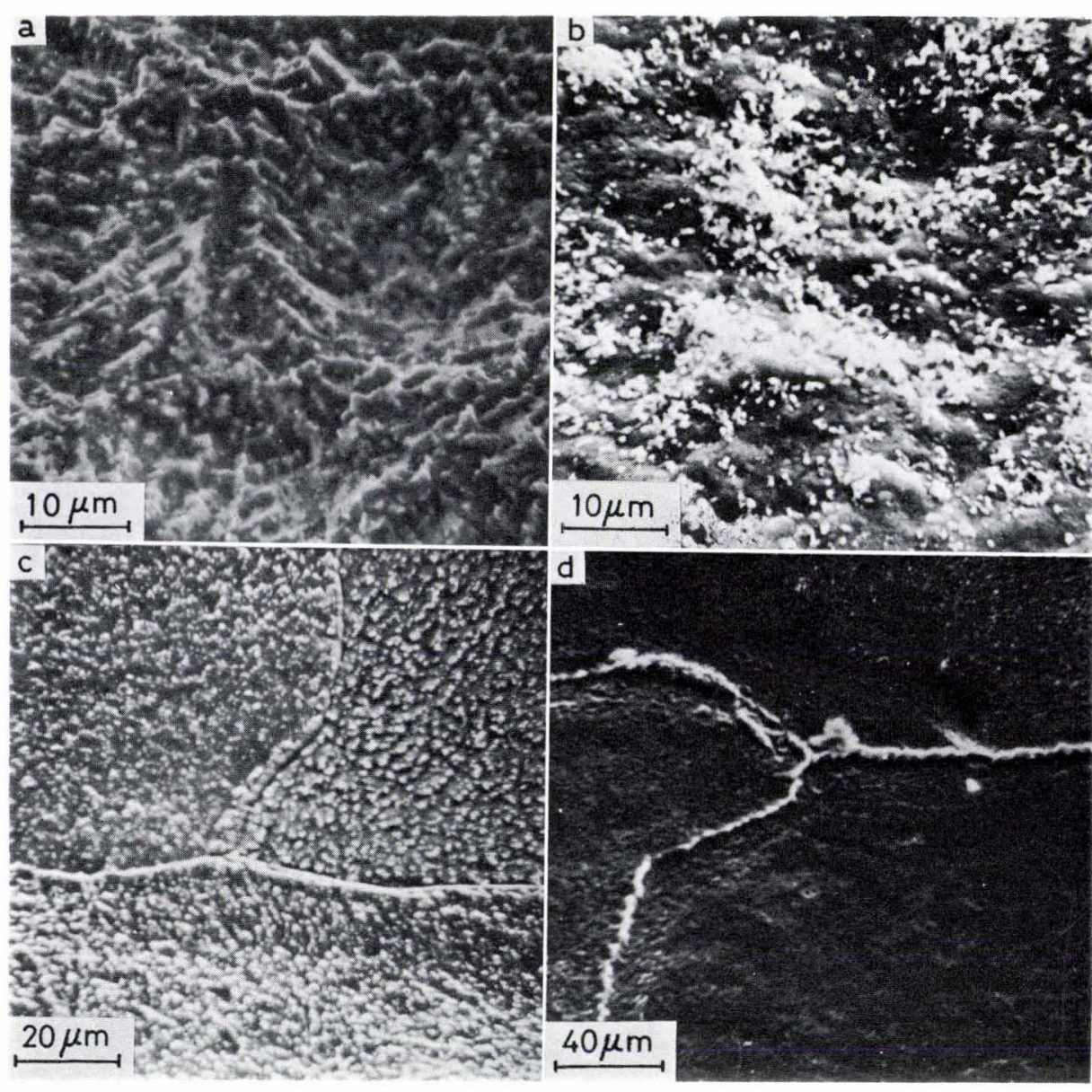

Photo. 3.

Substrate surfaces after $1000 \mathrm{~min}$ oxidation under (a) condition 1, showing a corrugated structure, (b) condition 2, oxide bridges, (c) condition 3 , slightly uneven surface, and (d) condition 4, pronounced ridges formed on grain boundaries of the substrate 
Photo. 4.

Fractured edges of oxide scales after 1000 min oxidation under (a) condition 1, showing columnar wustite and magnetite layers, and (b) condition 4, equiaxed wustite and magnetite layers
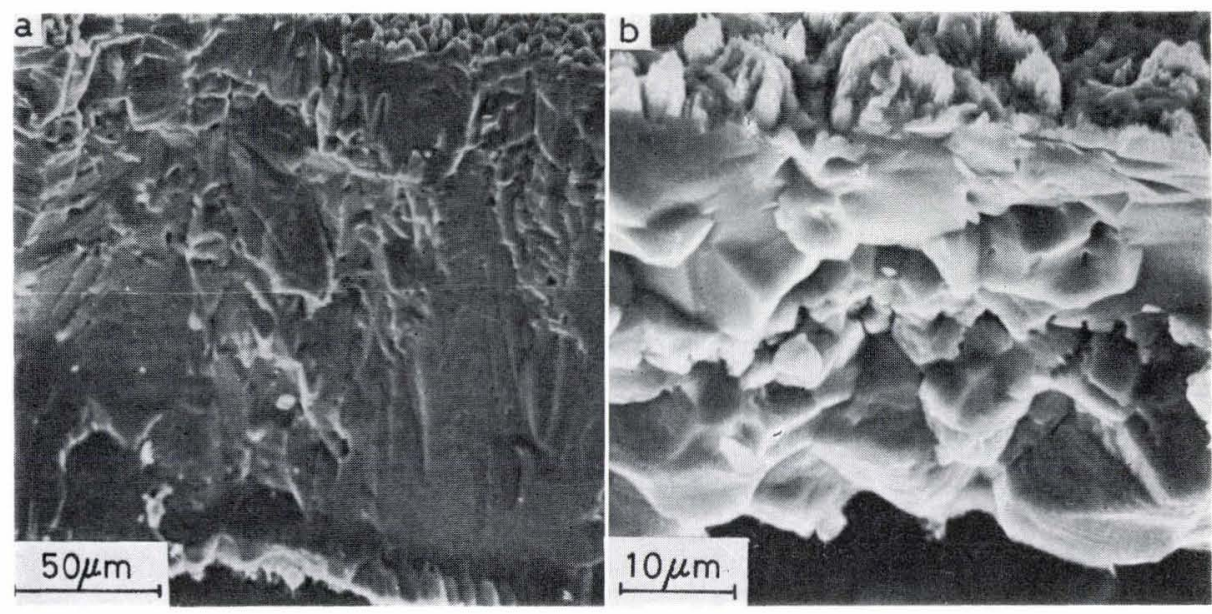

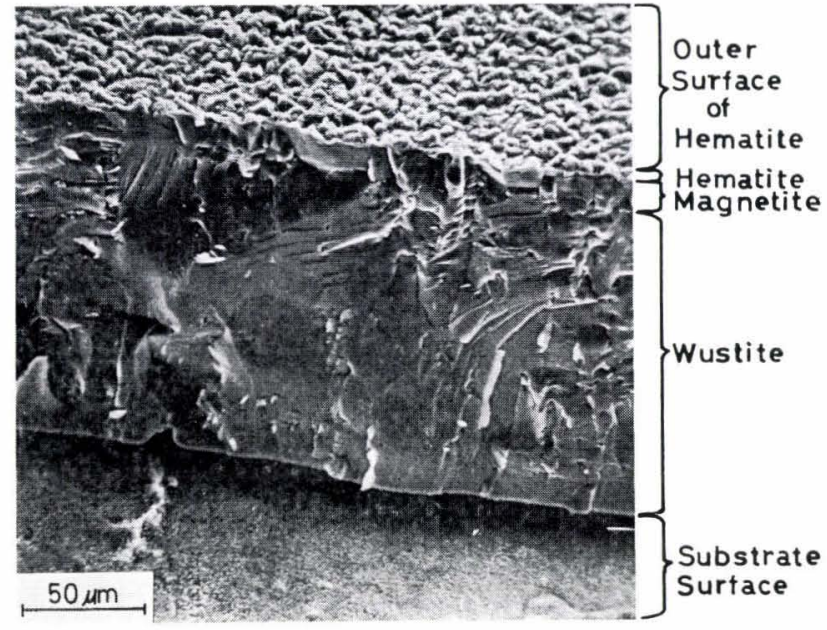

Photo. 5. Features of substrate surface and fractured edge of oxide scale after 300 min oxidation under condition 5

This is almost the case for the oxidation under condition $\mathbf{l}$ in the present study. However, abrupt decreases in oxidation rates were observed after the initial nearly parabolic periods under conditions 2 to 4 . This may be accounted for by the occurrence of partial separations of the oxide scales from the substrates over appreciable areas, since the partial separation decreases the contacting area through which mass transport from iron substrate to the scale is maintained.

This view may be supported by the observation that the oxide scales formed under these conditions were poorly adherent to the substrates and the existence of both oxide bridges, Photo. 3 (b), and ridges which were formed on the grain boundaries of the substrate surface, Photo. 3 (d). These oxide bridges and ridges may be the paths for the mass transport after the extensive partial separation took place. Similar oxide bridges were observed ${ }^{20)}$ when the rate of oxidation dropped abruptly in a manner similar to that shown during oxidation under condition 2 .

Even after the partial separation takes place at the wustite/iron interface, stresses are still developed, since conversion of the lower oxides to the higher oxides continues involving volume changes at the two solid/solid interfaces.
The transformation of columnar wustite to an equiaxed structure and the decrease in relative thickness of the wustite layer, Photo. 4 (b), further support the above view, since they have been reported ${ }^{7)}$ to take place during periods after the partial separation. The driving force for the partial separation has been reported $^{7)}$ to be void formation at the scale/substrate interfaces by the coalescence of excess vacancies or stresses developed during oxidation or, possibly, a combination of both.

The effect of the stresses may not be simple, since it may act to enhance the partial separation by, for instance, blistering once sites for stress concentration are formed, while compressive stresses developed by the mechanisms (a) and (b) may act to improve the adherence by inducing plastic flow of wustite toward the interfacial voids. This results in the elimination of the voids.

Before starting the direct comparison of unilateral and bilateral oxidation, it may be reasonable and convenient to clarify effects of other metallurgical pretreatments, since several pre-treatments were introduced prior to oxidation.

\section{Effect of Cold Work and Full Annealing on Oxidation}

Effects of cold work on the oxidation behaviour of a range of metals have been studied by several workers, ${ }^{27-29)}$ who have reported that cold-worked specimens oxidized more rapidly than unworked specimens under identical conditions. More recently Carpenter and Ray. ${ }^{20)}$ have observed that cold-rolled Remko iron coupons oxidized more rapidly than fully annealed specimens at $700^{\circ}$ and $800^{\circ} \mathrm{C}$. They attributed this not only to the additional vacancy sinks available in the cold-worked material to suppress both void formation and separation at the oxide/metal interface, but also to the enhanced diffusion through the oxide layer formed on cold-rolled iron. This increase in diffusion rate may be due either to a steeper vacancy concentration gradient resulting from the more efficient elimination of vacancies or to the numerous short-cut diffusion paths available on such a surface, or it may be that a combination of both effects may be involved.

The result of the present investigation again con- 
firmed the effect of cold rolling observed by Carpenter and Ray. (compare curves 4 and 5).

Their reasoning may apply to the present study, even recrystallization took place during the annealing at $700^{\circ} \mathrm{C}$ and the factors referred to above become less important with increase in oxidation time at high temperatures, since further recrystallization of the substrate takes place.

However, the grain size after this treatment was still much smaller than that of the fully annealed specimen. ${ }^{18)}$

Grain grow $\mathrm{th}^{30}$ ) of oxide may also take place at relatively high temperatures leading to a decreased contribution from short-cut diffusion paths for longer oxidation periods.

A further possible reason responsible for the observed differences is the fact that cold-rolled polycrystalline iron in general develops a texture ${ }^{31)}$ mainly composed of (100) planes or of (100) and (111) planes, while annealing treatments mainly composed of (111) planes and proportion of this plane increases as annealing temperature rises. ${ }^{31,32)}$ On the other hand, the rate of nucleation of iron oxide on (111) planes has been reported ${ }^{33)}$ to be one order of magnitude smaller than that on (100) planes. Thus, the difference in rates of oxidation during the initial periods between recrystallized (curves 1 and 2) and fully annealed (curves 3 and 4) specimens may be accounted for by the factors shown above.

\section{Effect of Annealing in a Mixture of Nitrogen-5\% Hydrogen}

Distinct differences in rates of oxidation between cold-rolled specimens and cold-rolled specimens followed by annealing treatment in a mixture of nitrogen $-5 \%$ hydrogen at $700^{\circ} \mathrm{C}$ for $1 \mathrm{~h}$ may be explained in terms of the effect of hydrogen anneal, since a similar decrease in the rate of oxidation was observed when the same specimens were treated in dry hydrogen prior to oxidation at $700^{\circ} \mathrm{C}$ by Carpenter and Ray. ${ }^{20)}$

Swanson and Uhlig' ${ }^{21)}$ have also observed that heat treatment in reducing hydrogen-water vapour mixtures modified oxidation rates of iron by developing surface facets of the substrate metal having an orientation corresponding to the minimum metal surface energy.

The solubility of hydrogen in iron at $700^{\circ} \mathrm{C}$ has been reported ${ }^{34)}$ to be $2 \times 10^{-4} \mathrm{wt}^{\%} \%$. This value may be too small to expect that the dissolved hydrogen affects the adherence of the scale to the substrate by forming gaseous products.

\section{Comparison of Unilateral and Bilateral Oxidation Behaviours}

This has been carried out by several investigators.

Tylecote ${ }^{35)}$ for example, has found that oxide scales formed on iron by unilateral oxidation were more adherent than those by bilateral oxidation, while Appleby and Tylecote. ${ }^{36)}$ have reported no difference between the rates of unilateral and bilateral oxidations of copper.
However, the results of the present investigation showed significant differences. A visual inspection after the unilateral oxidation revealed that all specimens were bent in such a way that the originally uncoated iron surfaces were convex outwards. This was in agreement with the result of stress measurement carried out by the present authors ${ }^{16)}$ who observed that tensile stresses were developed during short periods ( 10 to $30 \mathrm{~min}$ ) and compressive stresses prevailed for the longer periods for the recrystallized specimens, while only compressive stresses were developed after $50 \mathrm{~min}$ oxidation of the fully annealed specimens.

On the other hand, there was no indication of bending in the bilaterally oxidized specimens of both recrystallized and fully annealed. Therefore, it is clear that the unilateral oxidation created bending moment.

Further, this moment is a net result of stresses arising from the possible mechanisms (a) to (c), and can readily induce the plastic flow of the scale.

Compressive stresses developed in wustite layer by mechanisms (a) and (b) may be exclusively responsible for the plastic flow of it.

Both the above fact and the morphological examination may allow the following possible explanation for the observed kinetic behaviour. The unilateral oxidation under condition 1 created a favourable situation for the plastic flow of the oxide scale resulting in good adherence of the oxide scale with the substrate. The enhanced plastic flow may have eliminated the interfacial voids, and thus maintained the high oxidation rate. The stress created during the rapid oxidation again enhanced the plastic flow of the oxide scale.

The corrugated surface of the substrate, Photo. 3 (a), may be a result of the plastic flow of the oxide scale together with the substrate. This structure is in good contrast with the substrate of the bilaterally oxidized specimen, Photo. 3 (b).

These processes may have continued untill the oxide scale reach a certain thickness where the rate of stress generation was not high enough to create significant plastic flow of the oxide scale or that the relatively less plastic hematite ${ }^{37)}$ and magnetite ${ }^{37)}$ layers grew to thickness large enough to resist the plastic flow of the wustite layer.

The enhanced plastic flow of the oxide scale by the unilateral oxidation may have masked the effect of pre-treatment in the nitrogen-5\% hydrogen mixture, while this pre-treatment resulted in a significant decrease in the oxidation rate after an initial period for the bilateral oxidation under condition 2 .

On the other hand, the unilateral oxidation increased the oxidation rate to a smaller extent for the fully annealed specimens. This is probably due to the different nature ${ }^{27,38)}$ of the oxide formed on the fully annealed specimen and also to the possibility that the rate of stress generation during the initial periods of the unilateral oxidation was insufficient to create plastic flow of the oxide scale which would tend to eliminate the interfacial voips. 
The observed differences in oxidation behaviours between under condition 5 and under conditions 2 and 4, though they all are bilateral oxidations, may be accounted for by the following consideration. Stresses developed during oxidation under condition 5 are sufficient to allow plastic flow of wustite eliminating the interfacial voids, while those under conditions 2 and 4 are insufficient because of different natures of oxides formed and substrates as has been indicated in $I V .1$ and 2.

This signifies that different natures of oxides formed on substrate of different conditions can modify the subsequent oxidation behaviours even weight gains during initial periods are similar.

It may be of importance to note that state of stresses present in oxide scales will modify the diffusion process through the layer and therefore, the oxidation kinetics in cases where the diffusion process is the rate determining step as suggested by Sartell et al. ${ }^{39)}$ The changed thickness ratio of wustite to magnetite layers, though the scale was adherent, as shown in Photo. 1 may be a result of stress relief by the unilateral oxidation, or at least a result of modified stress system in the scale.

This view is emphasized by the observation that the above ratios are different between oxidation under conditions 1 and 5, even though the weight gains are similar after 1000 min oxidation.

\section{Summary}

Rates of unilateral oxidation were generally higher than those of bilateral oxidation for the thin coupon specimens after the specified pre-treatments. This has been considered to be due to the bending moment created by the unilateral oxidation favouring the plastic flow of the oxide scales which eliminated the interfacial voids or prevented the void formation at the scale/substrate interfaces during oxidation.

However, the above effect was not so significant for the fully annealed specimens. Different growth characteristics of oxide and the slower rate of oxidation, thus slower rate of stress generation, during the initial periods may be responsible for this.

It should be emphasized that the stress system established during the initial periods has a significant influence upon the subsequent oxidation behaviour, even though the weight gains are similar during the initial periods.

Furthermore, it is suggested that a modification of stress state in the growing oxide scale may change the diffusion process across it resulting in a different scale morphologies and hence kinetic behaviour.

\section{Acknowledgements}

The authors would like to express their thanks to Professor H.K.M. Lloyd of University College, Cardiff, for provision of the research facilities.

\section{REFERENCES}

1) R. F. Tylecote: J. Inst. Metals, 78 (1950-51), 301.

2) R. F. Tylecote: JISI, 196 (1960), 135.

3) R. F. Tylecote and T. E. Mitchell: JISI, 196 (1960), 445.

4) D. L. Douglass: Oxid, Met., 1 (1969), 127.

5) J. Stringer: Corros. Sci., 10 (1970), 513.

6) R. Hales and A. C. Hill: Corros. Sci., 12 (1972), 843.

7) S. Taniguchi and D. L. Carpenter: Submitted to Trans. $J M$.

8) G. W. Greenwood and J. E. Harris: Acta Met., 13 (1965), 936.

9) J. D. Watchman, Jr.: Bull. Am. Ceram. Soc., 46 (1967), 756.

10) R. E. Pawel, J. V. Cathcart and J. J. Campbell: J. Electrochem. Soc., 110 (1963), 551.

11) R. E. Pawel and J.J. Campbell: Acta Met., 14 (1966), 1827.

12) R. E. Pawel and J.J. Campbell: J. Electrochem. Soc., 116 (1969), 828.

13) V. R. Howes and C. N. Richardson: Corros. Sci., 9 (1969), 385.

14) A. Norin: Oxid. Met., 9 (1975), 259.

15) G. B. Gibbs, M. R. Wootton, W. R. Price and K. E. Hodgson: Oxid. Met., 7 (1973), 185.

16) S. Taniguchi and D. L. Carpenter: Trans. ISIJ, 18 (1978), 530 .

17) G. Gutzeit: Symp. Electroless Nickel Plating, ASTM Spec. Pub., (1959), No. 265, 3.

18) S. Taniguchi: Ph.D. Thesis, University of Wales, (1976).

19) W. H. Metzger Jr.: Symp. Electroless Nickel Plating, ASTM Spec. Pub., (1959), No. 265, 13.

20) D. L. Carpenter and A. C. Ray: Corros. Sci., 13 (1973), 493.

21) A. W. Swanson and H. H. Uhlig: J. Electrochem. Soc., 121 (1974), 1551

22) O. Kubaschewski and B. E. Hopkins: Oxidation of Metals and Alloys, 2nd ed., Butterworth, London, (1967).

23) A. C. Ray: Ph.D. Thesis, University of Wales, (1971).

24) M. H. Davies, M. T. Simnad and C. E. Birchenall: J. Metals, 3 (1951), 889.

25) J. Paidassi: Acta Met., 6 (1958), 184.

26) B. W. Dunnington, F. H. Beck and M. G. Fontana: Corrosion, 8 (1952), 2.

27) S. I. Ali and G. C. Wood: J. Inst. Metals, 97 (1969), 6.

28) D. Caplan and M. Cohen: Corros. Sci., 6 (1966), 321.

29) D. Caplan, G. I. Sproule and R. J. Hussey: Corros. Sci., 10 (1970), 9.

30) K. N. Strafford: Werkstoffe Korrosion, 23 (1972), 755.

31) S. Nagashima: Shugososhiki, Kinzokugaku-Shinsho, Japan Inst. Metals, (1966).

32) R. L. Whiteley and D. E. Wise: Flat Rolled ProductsIII, Met. Soc. Conf., XVI, edited by E. W. Earhart Interscience Publishers, New York, (1962).

33) J. Bardolle and J. Benard: Compt. Rend., 239 (1954), 706.

34) M. Hansen: The Constitution of Binary Alloys, 2nd ed. New York, (1958).

35) R. F. Tylecote: Mem. Sci. Rev. Met., 62 (1965), 241.

36) W. K. Appleby and R. F. Tylecote: Corros. Sci., 10 (1970), 325.

37) J. D. Mackenzie and C. E. Birchenall: Corrosion, 13 (1957), 17.

38) A. Ronnquist and K. Thomas: Bril. Corrosion J., 1 (1965), 47.

39) J. A. Sartell, R.J. Stokes, S. H. Bendel, T. L. Johnston and C. H. Li: Trans. AIME, 215 (1959), 420. 